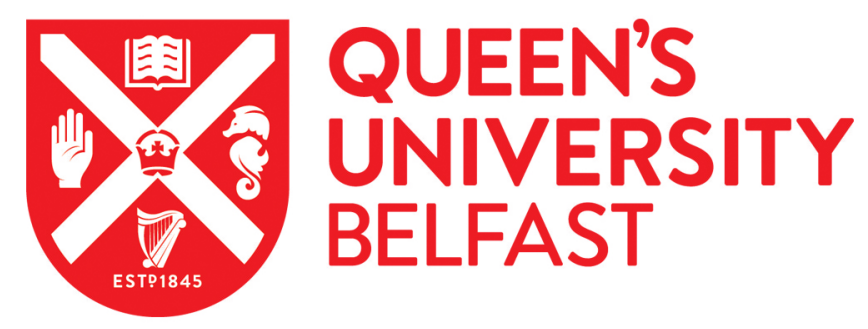

\title{
Physical Layer Secure Communication Using Orbital Angular Momentum Transmitter and a Single-Antenna Receiver
}

Abbasi, M. A. B., Fusco, V., Naeem, U., \& Malyuskin, O. (2020). Physical Layer Secure Communication Using Orbital Angular Momentum Transmitter and a Single-Antenna Receiver. IEEE Transactions on Antennas and Propagation. https://doi.org/10.1109/TAP.2020.2981732

Published in:

IEEE Transactions on Antennas and Propagation

Document Version:

Peer reviewed version

Queen's University Belfast - Research Portal:

Link to publication record in Queen's University Belfast Research Portal

Publisher rights

(c) 2020 IEEE.

This work is made available online in accordance with the publisher's policies. Please refer to any applicable terms of use of the publisher.

\section{General rights}

Copyright for the publications made accessible via the Queen's University Belfast Research Portal is retained by the author(s) and / or other copyright owners and it is a condition of accessing these publications that users recognise and abide by the legal requirements associated with these rights.

Take down policy

The Research Portal is Queen's institutional repository that provides access to Queen's research output. Every effort has been made to ensure that content in the Research Portal does not infringe any person's rights, or applicable UK laws. If you discover content in the Research Portal that you believe breaches copyright or violates any law, please contact openaccess@qub.ac.uk. 


\title{
Physical Layer Secure Communication using Orbital Angular Momentum Transmitter and a Single Antenna Receiver
}

\author{
M. Ali Babar Abbasi, Member IEEE, Vincent Fusco, Fellow, IEEE, \\ Umair Naeem, Member, IEEE, and Oleksandr Malyuskin, Member, IEEE
}

\begin{abstract}
Orbital Angular Momentum (OAM) for radio communications has the potential of simultaneously transmitting multiple signals at the same frequency and time resources. This multiplies the achievable channel capacity at a given bandwidth by increasing the available number of simultaneous data streams. One downside of OAM radio communication is the requirement of multi-mode radio frequency ( $R F$ ) hardware at both ends of a link i.e. transmitter as well as the receiver. This is not always practically viable, especially as we move towards low profile receivers in future communication devices. In this work, we present a novel method of OAM-based radio communication with enhanced physical layer security that requires only a single antenna receiver. We first present the system architecture, then we design and realize a Rotman lens-based circular antenna array transmitter operating at $5.8 \mathrm{GHz}$. We then experimentally verify the capability of the hardware to create multiple modes. As a proof-of-concept, we propose a communication system that simultaneously uses mode 0 and +1 of the OAM beamformer and in doing so show how a single receive antenna can be used for data recovery. We first identify a general analogue modulation expression and use the proposed system to transmit digitally modulated data stream to a single antenna equipped receiver. A pre-communication training sequence is required to realize the proposed approach. Experimental results verify the simulated predictions.
\end{abstract}

Index Terms - Antenna, antenna array, Orbital Angular Momentum, Rotman, Lens, Secure communication, physical layer security.

\section{INTRODUCTION}

$\mathrm{T}$ $\mathrm{HE}$ radio frequency spectrum is getting congested every year, especially the frequency band from $500 \mathrm{MHz}$ to 10 GHz. This band is used by many communication technologies including the Global System for Mobile Communications $(\mathrm{GSM}), 4^{\text {th }}$ and earlier generations of cellular communication, sub-6 GHz 5G, wireless fidelity (Wi-Fi), and satellite comms. The demand for disruptive technologies that can utilize the available resources for communication has increased significantly. Orbital Angular Momentum (OAM) radio is under investigation currently as one such technology, because

Manuscript received XXX, revised XXX, published XXX. This work was supported by the UK Engineering and Physical Science Research Council (EPSRC) under Grant EP/P000673/1 and EP/N020391/1.

M. A. B. Abbasi,V. Fusco, U. Naeem and O. Malyuskin are with The Centre for Wireless Innovation (CWI), The Institute of Electronics, Communications of some of its promising features that are not available in a standard radio [1]. The most important property of OAM is its utilization of the same time and frequency resources for orthogonal data transmission. Several studies have highlighted novel approaches to achieve OAM wave-fronts. These include, but are not limited to, metasurface based [2]-[4], reflector-array based [5], ceramic antenna array [6], patch antenna array [4], [7] and helical antenna based [8] OAM transmitters.

At the transmitter side of an OAM link, generally, an antenna with a feed network generates two (as in [9]) or higher number of independent modes [8], [10]. This is generally achieved by fixed phase shifter-based feed networks. Some researchers also introduced reconfigurability into the feed network to support more sophisticated modes [4], [11]. Free space radio communication has also been shown using Rotman lens as feed network for mode separation as demonstrated in [12], [13]. A recent study has also shown an OAM wave-front formation without the requirement of any feed network [6].

At the receiver end, sometimes hardware analogues to the transmitter radio is used to successfully decode the transmitted signal, for example in circular array-based OAM reception in [14]-[17] and in the planar array system [18]. Receive-side methods for OAM signal recovery are problematical. Solutions include OAM phase properties decoded at a long-distance reception with the help two electrically large antennas [19]. Another study explained a new Doppler-based directionfinding approach [20] that detects individual OAM modes based on an interpolation technique.

One of the key bottlenecks in the successful realization of OAM communication is the position of nulls at the boresight of transmitter antenna array [3], [5], [9]-[11], [21], [22]. This limits the coverage area to a doughnut-shape sector especially when we look at the radiation of higher modes. One approach is to use lenses to collimate the beams [23], [24]. In addition to this, the literature has repeatedly shown the distinction between the received signal power densities at the receiver antenna when different modes are used to transmit orthogonal signals. This creates unequal power at radio receivers which sometimes leads

and Information Technology (ECIT), School of Electronics, Electrical Engineering and Computer Science (EEECS), Queen's University Belfast, Belfast BT3 9DT, United Kingdom (email: m.abbasi@qub.ac.uk, v.fusco@ecit.qub.ac.uk,u.naeem@qub.ac.uk, o.malyuskin@qub.ac.uk) 


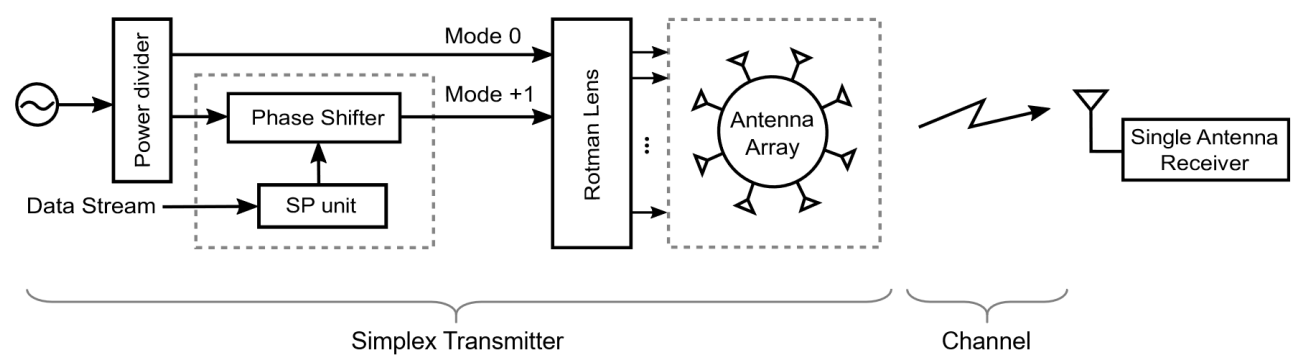

Fig. 1. Hardware block diagram of the OAM communication system with enhanced physical layer security. Operation of the simplex transmitter is provided in Table I.

to transmitted signal been undetected. In other words, signal-tointerference-plus-noise ratio's (SINR) dynamic ranges for the received signal at one mode sometimes dominate the signal in other modes which can result in information loss.

In this paper, we propose a novel mode-mixing OAM transmitter topology using a phase modulation (PM) approach which requires a comparatively simple system at the receiver to decode the transmitted information. The power density range of the transmitted signal is constrained within the detectable range of the receiver by simultaneously exciting multiple modes. The approach also offers a direction dependent secure signaling scheme which does not require additional data payload, thereby adding a level of physical layer security to a simplex communication link. As a proof-of-concept, we use modes 0 and +1 of a Rotman lens-based OAM transmitter and successfully recover a 4-QPSK data stream using a far-field positioned single antenna equipped receiver. Unlike in [20] where OAM detection is done based on the Doppler effect in the frequency domain, this work is purely signal processing based utilizing the demodulated output at a single antenna receiver.

Section II of the paper presents the system block diagram and explains the proposed approach, Section III discusses the hardware realization and experimental results of a circular array of patch antenna fed by Rotman lens mode generator. Section IV discussed the mode-mixing capabilities based on the experimental data. Section V discusses physical layer secure communication example. Section VI discusses the shortcomings of the approach where findings are concluded on section VII.

\section{SySTEM MOdEL}

Consider the mode-mixing OAM transmitter topology in Fig. 1. Here mode 0 and +1 (also referred to as $l=0$ and $l=+1$ ) of an OAM circular array transmitter are simultaneously excited by a frequency source and an unequal power divider. The main reason for using unequal power is to balance multiple modes (this ratio is 3:1 for the example presented in section III). Mode 0 is excited directly while a variable full-cycle phase shifter, typically voltage control, is added before excitation input of mode +1 . The phase difference induced within mode +1 of the OAM transmitter is controlled by a signal processing unit, which takes in the information stream that is intended to be transmitted. To elaborate on this, we first build our analysis based on classical analogue modulation. We then use this analysis to assist in designing a digital modulation scheme. A standard analogue PM signal can be written as:

$$
i_{\mathrm{PM}}(t)=A_{c} \sin \left[\omega_{c} t+m_{p} \sin \omega_{m} t+\varphi\right]
$$

where, $m_{p}=K_{p} A_{m}$ is the modulation index when $K_{p}$ is the proportionality constant for PM. $\varphi$ is the time-independent phase offset. For simplicity, we consider $\varphi=0^{\circ}$. Subscript $p$ in $m_{p}$ shows that the modulation index is for PM waveform. $\theta_{\mathrm{PM}}$ is the instantaneous phase of the carrier defined by

$$
\theta_{\mathrm{PM}}=\omega_{c} t+m_{p} \sin \omega_{m} t
$$

when modulated signal and the carrier signal is $e_{m}=A_{m} \cos \omega_{m} t$ and $e_{c}=A_{c} \cos \omega_{c} t$ respectively. Here $A$ is the magnitude and $\omega$ $=2 \pi f$ for the frequency $f$ while subscripts $c$ and $m$ represent carrier and modulated signal respectively. The expression of the modulation vector for (2) can be written as:

$$
i_{\mathrm{PM}}(t)=A_{c}\left[\begin{array}{l}
J_{0}\left(m_{p}\right) \sin \omega_{c} t \\
+J_{1}\left(m_{p}\right)\left[\sin \left(\omega_{c}+\omega_{m}\right) t-\sin \left(\omega_{c}-\omega_{m}\right) t\right] \\
+J_{2}\left(m_{p}\right)\left[-\sin \left(\omega_{c}+2 \omega_{m}\right) t+\sin \left(\omega_{c}-2 \omega_{m}\right) t\right] \\
+J_{3}\left(m_{p}\right)\left[\sin \left(\omega_{c}+3 \omega_{m}\right) t-\sin \left(\omega_{c}-3 \omega_{m}\right) t\right] \\
+\cdots
\end{array}\right]
$$

when $J_{n}(x)$ is the first kind Bessel function of the order $n$ [25]. On a base-band frequency spectrum, each summation term in (3) represents a separate sideband. For the majority of the signals, the baseband bandwidth of PM is considered to be $\sim 2\left(\Delta \theta_{\mathrm{PM}}+1\right) f_{m}$ where $\Delta \theta_{\mathrm{PM}}$ is the peak phase deviation in radians. To understand the impact of $\theta_{\mathrm{PM}}$ on the radiated signal, it is important to analyze the radiation performance of an OAM transmitter.

\section{OAM TRANSMITTER REALIZATION}

To achieve OAM transmission, we built a Rotman lens-based circular antenna array [13]. The Rotman lens is designed on Taconic RF-60 substrate operating at $f_{c}=5.8 \mathrm{GHz}$. The design parameters of the lens main body were set as: on-axis focal length $l_{l}=4.5 \lambda$, focal angle $\alpha=30^{\circ}$, on-axis to off-axis focal 


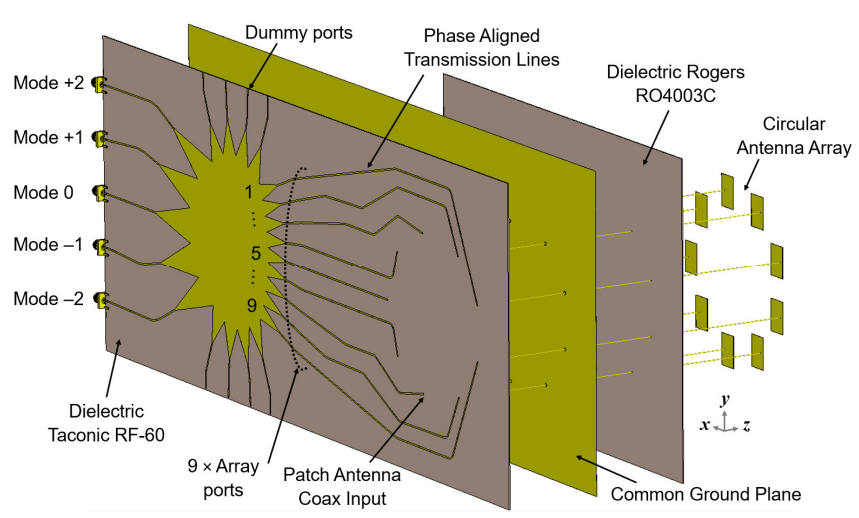

(a)

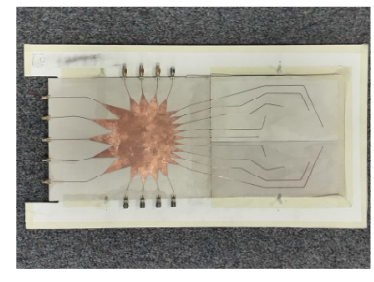

(b)

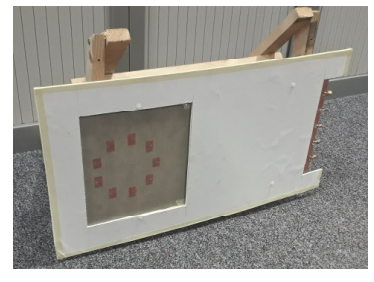

(c)
Fig. 2. (a) Geometrical configuration of multi-mode OAM transmitter. Taconic RF-60 sheet dimensions are $477 \times 198 \mathrm{~mm}^{2}$, dielectric constant $\left(\epsilon_{r}\right)$ is 6.15 , loss tangent $(\tan \delta)$ is 0.0038 and thickness $(h)$ is $635 \mu \mathrm{m}$. Rogers RO4003 dimensions are $274 \times 198 \mathrm{~mm}^{2}, \epsilon_{r}=3.38, \tan \delta=0.0027$ and $\mathrm{h}=508 \mu \mathrm{m}$. Photographs of (b) back side and (c) front side of the circular antenna array.

lengths ratio $\beta=0.88$, expansion factor $\gamma=\sin (\rho) / \sin (\alpha)=0.9$, when $\rho$ is the sweep angle. The Rotman lens has 5 beam ports, 9 array ports and 8 dummy ports. SMA connectors are mounted at the RF-in i.e at the beam port side. The array port side is connected to 9 phased aligned transmissions lines leading to each of the antenna unit cells in the circular array of patch antennas. All the dummy ports are terminated to a $50 \Omega$ matched load. Further details on design guidelines for the Rotman lens can be found in [26]. The antenna array unit cell dimensions are $13.20 \times 20 \mathrm{~mm}^{2}$ and the coax feed is located $3.10 \mathrm{~mm}$ inside the patch from the patch's broader side. $9 \times$ patch antenna unit cells form a circular array having a diameter of $\sim 105 \mathrm{~mm}$ which is around $2 \lambda$. For mode +1 , the diameter of the circular array is directly proportional to the peak gain and inversely proportional to the zenith angle at the maximum gain point (details can be found in [27]). The selected diameter $2 \lambda$ provides a relative trade-off between peak gain and the zenith angle. The schematic of the OAM transmitter is shown in Fig. 2(a). Note that the antenna orientation along the $y z$-plane of the patch antennas connected to beam ports $\{1,2,8,9\}$ is mirrored compared to the patch antennas connected to array ports $\{3,4,5,6,7\}$. Fabrication of all the layers was done using an LPKF Protomate H100 milling machine. Fig. 2(b) shows the fabricated Rotman lens layer while Fig. 2(c) shows the OAM transmitter mounted to a non-metallic frame for measurements. Simulated far-field radiation performance of the OAM transmitter is presented in Fig. 3. Excitation of the mode 0 port shown in Fig.2(a) results in a high directivity beam in the boresight direction. The simulated peak gain for this mode 0 excitation is $16.3 \mathrm{~dB}$. Mode +1 and +2 excitations reveal doughnut-shape directive beams with a null along the broadside direction with peak gain of 11.4 and $10.6 \mathrm{dBi}$. These are also
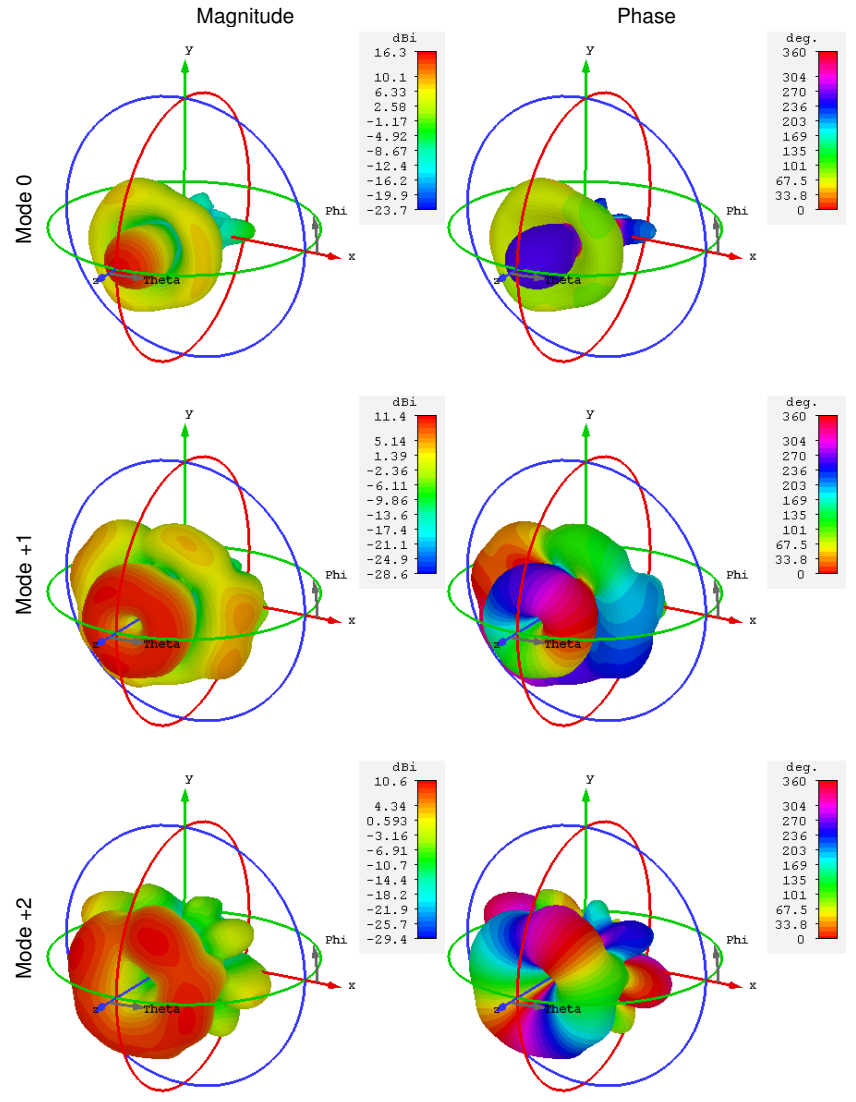

Fig. 3. Simulated far-field magnitude and phase of the circular array of patch antennas against multiple modes when RF-in is excited.

referred to as vortex beams [28]. A phase spiral wavefront with 1 cycle is evident for mode +1 excitation, and 2 cycles for mode +2 excitation in Fig, 3 simulated phase plots. This is also verified from the measured near-field phase plots presented in Fig. 4. The simulated and measured phase contours agree well with each other for mode 0 and 1 , while there is a phase spiral discontinuity in the phase of mode +2 due to hardware anomalies that can be offset using the design approaches in [13]-[16], [29]. Since in this paper we consider mode 0 and +1 so the phase discrepancy in mode +2 is of no importance. Note that a regular convex lens structures and specialized metasurfaces can be used to collimate the beam (like ones presented in [23], [24], [29]). Mode purity of the OAM transmitter is evaluated using spatial spectrum method [30], [31], defined as the ratio between power in the dominant mode over the overall power in all the other modes, given by

$$
C_{l}=\frac{P_{l}}{\sum_{q=-\infty}^{\infty} P_{q}} .
$$

Where $l$ represents the mode number $(l=0, \pm 1, \pm 2 \ldots)$. Over the scanning plan of $300 \times 300 \mathrm{~mm}^{2}$, the simulated mode purity for mode 0 and +1 is $>90 \%$, while the evaluated mode purity using measured data is $74.75 \%$ and $61.38 \%$, for mode 0 and +1 respectively. The implications of these mode purity figures will be discussed in section $\mathrm{V}$. 


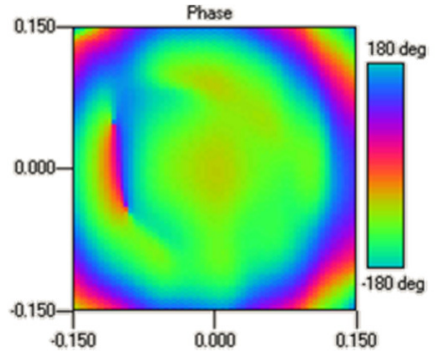

(a)

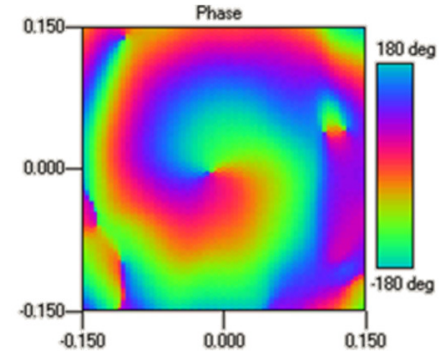

(b)

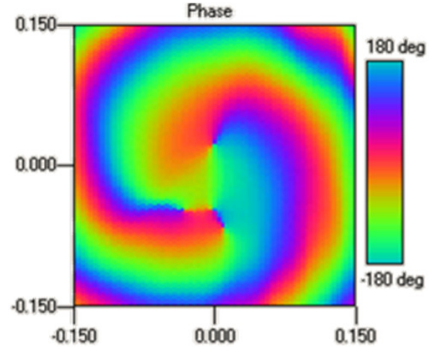

(c)

Fig. 4. Measured near-field phase of the circular array when (a) Mode 0, (b) Mode +1 and (c) Mode +2 are excited. 2-dimensional map axis represent the measuring probe position in meters in near-field anechoic chamber.

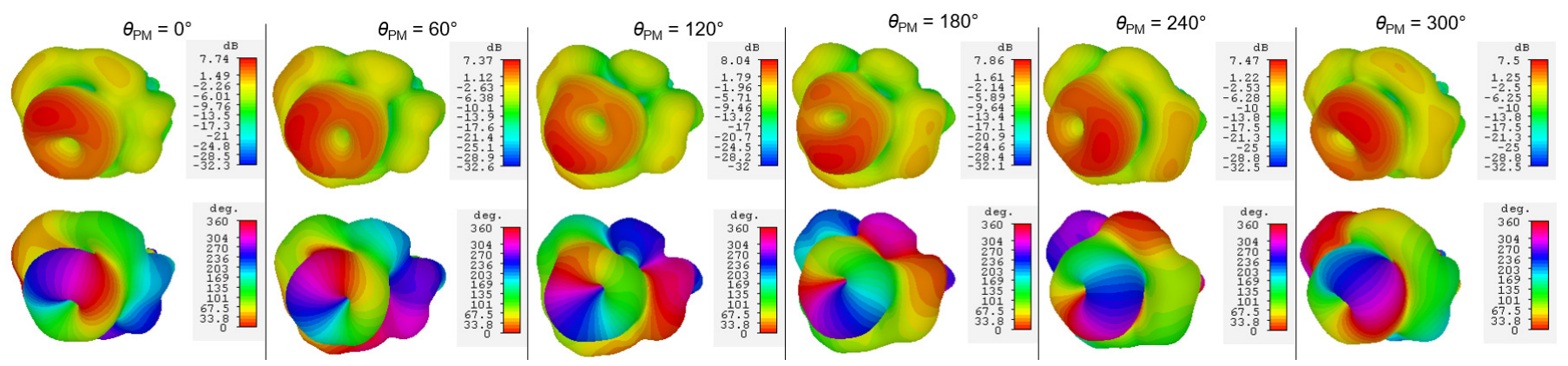

Fig. 5. Simulated far-field gain pattern of the OAM transmitter when $\theta_{\mathrm{PM}}$ is varied with step of $60^{\circ}$.

\section{MODE-MiXING OAM TRANSMITTER}

Let's continue the analysis we left in section II. The radiated signal envelop of an OAM transmitter will have an average farfield electric field $E_{c}$ at the $f_{c}$, and can be superimposed by a modulating signal of amplitude $K_{a} A_{m}$ with frequency $f_{m}$, thus,

$$
i(t)=E_{c}\left(\sin \omega_{c} t+K_{a} \sin \omega_{c} t \sin \omega_{m} t\right) .
$$

This represents analogue amplitude modulation (AM) and can also be written as

$$
i(t)=E_{c}\left(\sin \omega_{m} t+\frac{1}{2} K_{a} \cos \left(\omega_{c}-\omega_{m}\right) t-\frac{1}{2} K_{a} \cos \left(\omega_{c}+\omega_{m}\right) t\right),
$$

which, in contrast to (3), represent only one upper or lower sideband on the baseband frequency spectrum. The signal processing (SP) unit inducing RF phase shift sensitive to $\theta_{\mathrm{PM}}$ will be proportional to the excitation voltage induced at the input of the circular antenna array. If the excitation voltage varies sinusoidaly, the antenna current, as well as the radiated field phase, is going to be sinusoidal. We can mathematically represent this by

$$
i(t)=E_{c}\left(1+K_{a} \sin \omega_{m} t\right) \times \sin \left(\omega_{c} t+m_{p} \sin \omega_{m} t\right) .
$$

We now have a combination of amplitude and phase modulation. Time varying phase shift at the excitation side is responsible for the phase modulation and the spatial variation of the fields introduce the amplitude dependence, and this impact is multiplicative as in (7). General forms of the modulation vectors of this case are stated at the end of the page.

It is worth noticing that while realizing such a system, the phase information $\theta_{\mathrm{PM}}$ directly translates to the position of the far-field radiation maximum. Consider the case where $\theta_{\mathrm{PM}}$ is varied continuously from $0^{\circ}$ to $360^{\circ}$ (analogue modulation) representing maximum achievable $\Delta \theta_{\mathrm{PM}}$. This results in spinning of the field maximum as can be seen from the simulated results presented in Fig. 5. We verified this by inducing phase-shifted simultaneous mode excitation in the
Carrier

First sideband

Second sideband

Third sideband and so on.

$$
E_{c}\left[J_{0}\left(m_{p}\right) \sin \omega_{c} t+K_{a} J_{1}\left(m_{p}\right) \cos \omega_{c} t\right]
$$



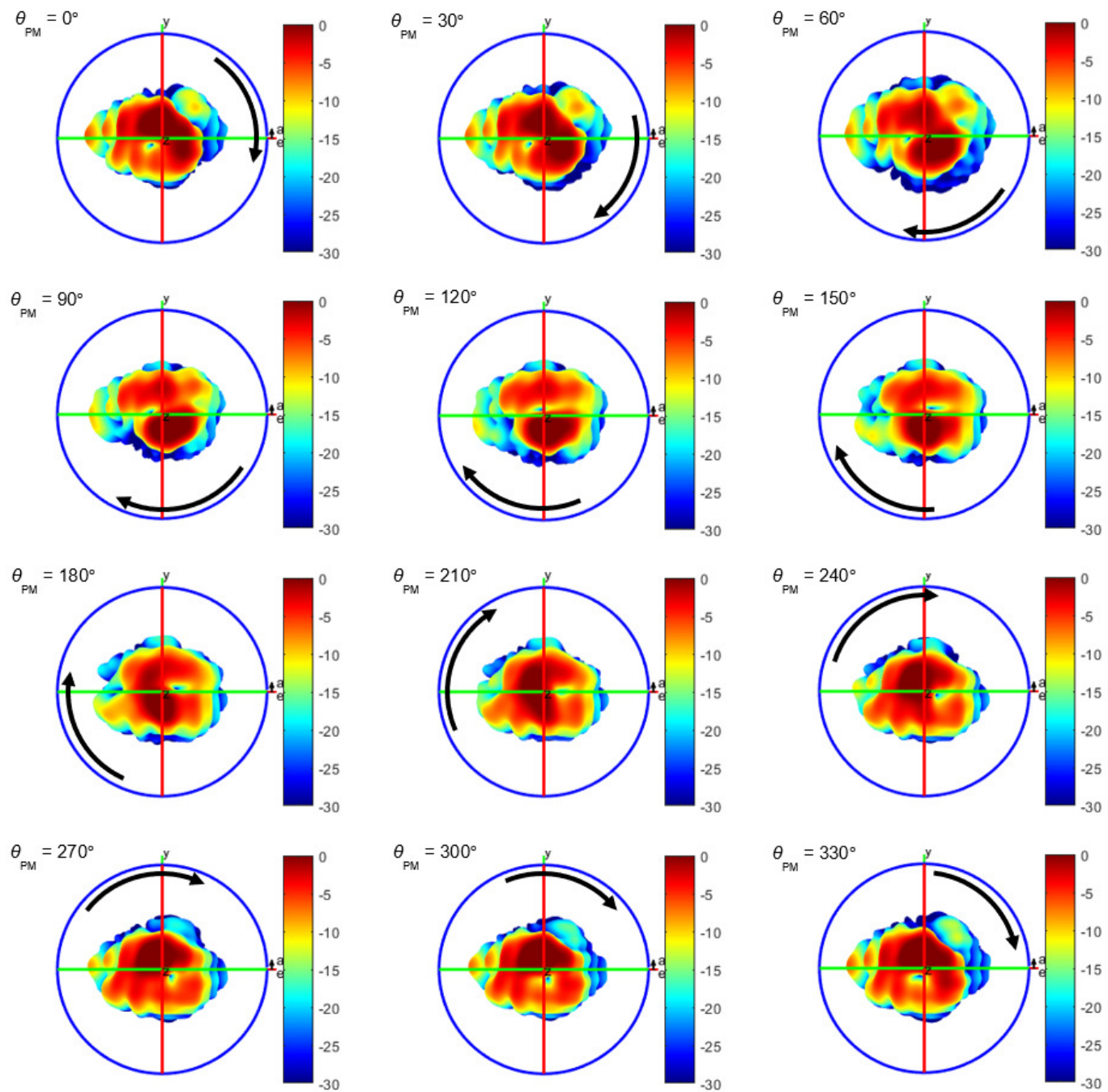

Fig. 6. Transformed far-field gain pattern using near-field measured data of the OAM transmitter.

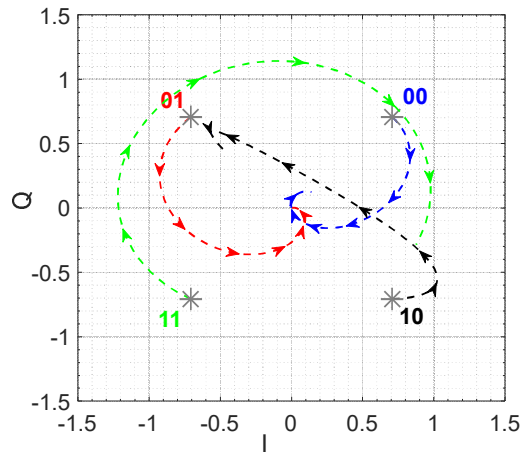

(a)

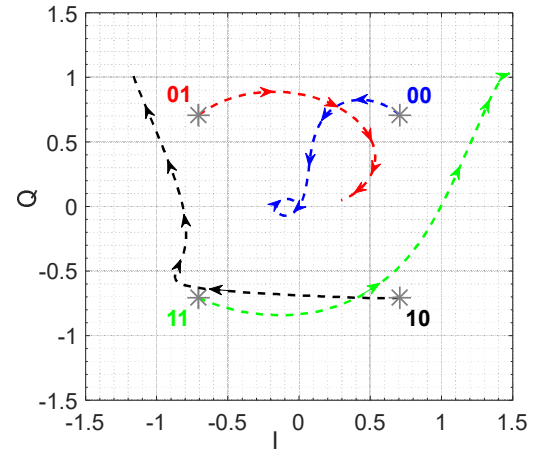

(b)

Fig. 7. The 4 color-coded symbols illustrate the expected ideal 4-QPSK constellation sectors while the same color-coded contours represent their travelling into neighbouring sectors when the single antenna receiver is moved away from reference position to (a) $+60^{\circ}$ and (b) $-60^{\circ}$ zenith angles

near-field chamber measurements of OAM transmitter hardware. We then used the two-dimensional fast Fourier transform (FFT) approach (see [32]) to compute the far-field plots as shown in Fig. 6. High-intensity colour code represents the peak power zone which covers almost all the $3 \mathrm{~dB}$ beamwidth area. The maximum field spinning along the boresight direction of the circular array can be clearly seen from the presented patterns as $\theta_{\mathrm{PM}}$ is ramped. Peak directional gain for the simulated cases varies from 12.9 to $13.5 \mathrm{dBi}$, i.e. the peak gain variation for all $\theta_{\mathrm{PM}}$ stays below $\sim 0.7 \mathrm{~dB}$. It is evident from Fig. 6, the spinning wavefront is not as well formed as was predicted by the simulated results in Fig. 5. This is due to fabrication anomalies in the hardware which eventually impacted the beam/mode purity of the OAM transmitter. In 


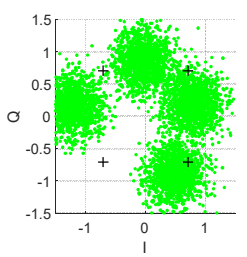

$-12^{\circ}$

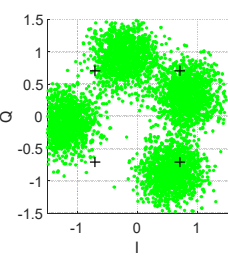

$-8^{\circ}$

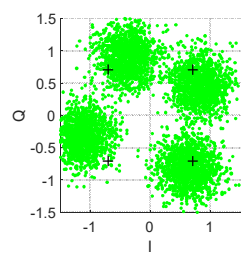

$-4^{\circ}$

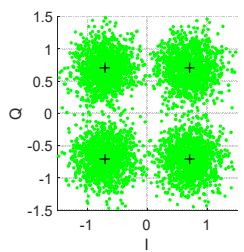

$0^{\circ}$

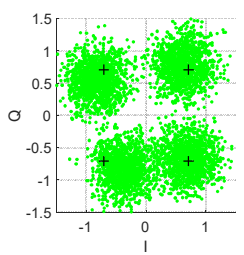

$4^{\circ}$

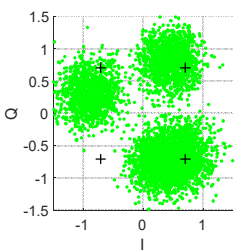

$8^{\circ}$

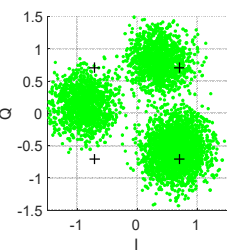

$12^{\circ}$

(a)

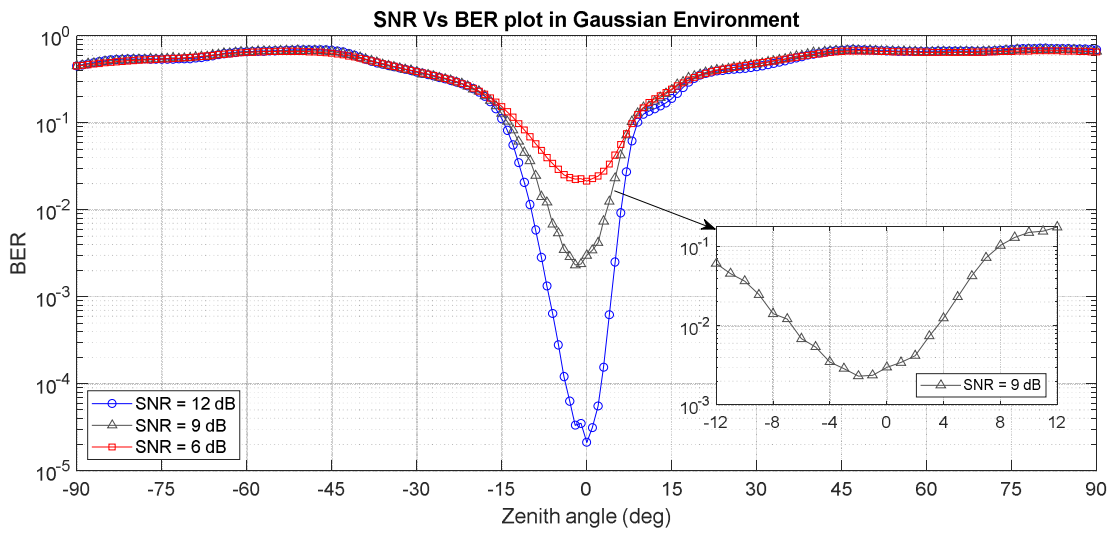

(b)

Fig. 8. (a) Received symbols by the single antenna receiver plotted on IQ constellation when it is moves along elevation. Plot at $0^{\circ}$ represents the reference receiver antenna position when $\mathrm{SNR}=9 \mathrm{~dB}$. (b) BER recorded at the receiver when it is moved from $-90^{\circ}$ to $90^{\circ}$ along elevation.

contrast to a standard OAM transmitter's spiral wavefront, i.e. only evident in the phase of the far-field patterns, our approach presents a spinning wavefront in the magnitude as well as the phase of the transmitter. This provides the benefit of direct amplitude-based reception of the signal which is possible using a single antenna receiver.

\section{SECURE COMmUnicAtion Using SingLE ANTENNA RECEIVER}

In this section, we present a simple digital modulation case study where the QPSK data stream is transmitted using the general architecture presented in section II and received by a single antenna receiver. A circular array of patch antennas is placed at the origin in the Cartesian coordinate system along $x y$ plane facing along the $+z$-axis when patch antenna dominant electric field vector is along $x$-axis. Mode-mixing OAM is active at $\theta_{\mathrm{PM}}=0^{\circ}$. An arbitrary receiver antenna is placed in the far-field along the field maxima of the OAM transmitter with matched polarization i.e. along $x$-axis, we consider this the reference position. Generally, in this situation, the receiver antenna should read an instantaneous magnitude and phase information as long as the received signal $\left(P_{R x}\right)$ at the single antenna receiver is higher than the noise $\left(P_{\text {noise }}\right)$ for all variations of $\theta_{P M}$. Let's consider the SP unit is encoding QPSK signal over $\theta_{\mathrm{PM}}$ such that $0^{\circ}$ to $360^{\circ}$ is quantized to represent 4 QPSK symbols. At a given instant, the received magnitude and phase information corresponding to each QPSK symbol is recoded and a point on an In-phase and Quadrature (IQ) constellation is plotted. For an ideal scenario when channel is noiseless, 4 symbols will represent ideal constellation points represented as a star in Fig. 7. If the receiver antenna is moved away from the reference position, the same point on the IQ constellation is bound to move away from the ideal QPSK since the magnitude and phase combination for the symbols have changed. Because the magnitude and phase information for antenna's reference position is unique, a new location of receiver antenna will scramble the constellation points.

To show this we use the measured data presented in Fig. 6 to first plot the ideal constellation. We then moved the receiver antenna from its reference position to $+60^{\circ}$ zenith angle in elevation (along $y z$-plane) and recorded the expected QPSK symbol contours. This is presented in Fig. 7(a). We then moved the receiver antenna to $-60^{\circ}$ zenith angle in elevation and again recorded the contours, that are shown in Fig. 7(b). In both cases, the symbol scramble is observed. In Fig. 7(a), constellation contours representing symbols 11 (green) and 01 (red) travelled through up to 3 neighboring sectors while symbol 00 (blue) travelled through all 4. A similar trend can be seen in Fig. 7(b) where all the contours travel through a minimum of 3 sectors as the single antenna receiver modes $-60^{\circ}$ zenith angles.

We further tested the limitations of the proposed system model by transmitting 10 million randomly selected bits via the mode-mixing OAM transmitter. This is done to ensure an accurate BER estimation i.e. up to $10^{-4}$. We computed the BER vs receiver antenna position when it is moved away from the reference, with a resolution of $1^{\circ}$, for $\mathrm{SNR}=6 \mathrm{~dB}, 9 \mathrm{~dB}$ and 12 dB. Clear visualization of the received signal scramble for 7 receiver positions are given in Fig. 8(a). Three conclusions are drawn from Fig. 8(a) and (b). First, the results show that proposed approach can ensure data reception in a desired direction. This enables a physical layer security that does not require complex receiver system. To further elaborate, examine 
TABLE I

COMMUNICATION WITH ENHANCED SECURITY USING OAM TRANSMITTER: OPERATION SUMMARY

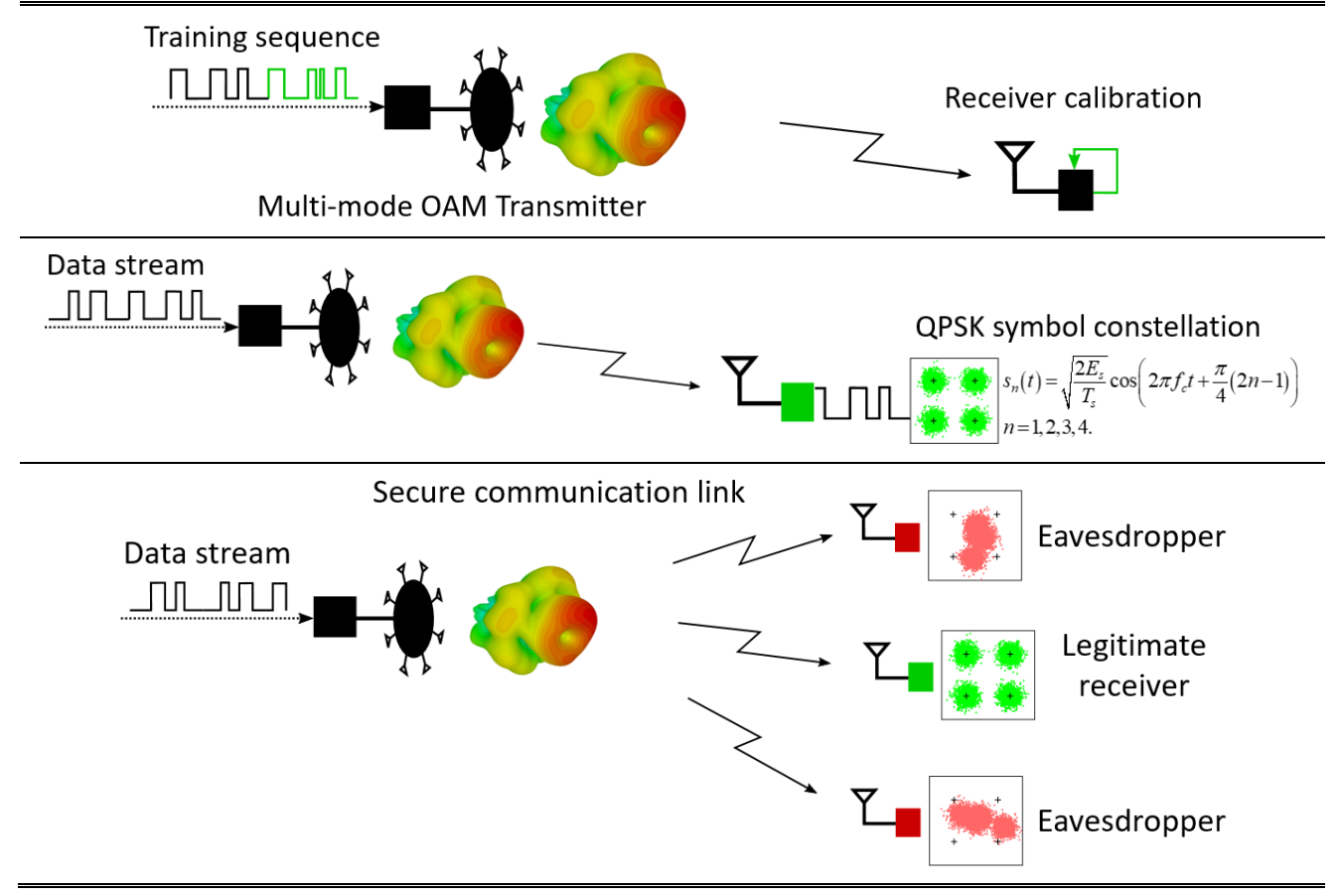

Fig. 8(a) when the single antenna receiver position is moving from $0^{\circ}$ to $12^{\circ}$. The received symbols on the constellation move away from their expected position along the prescribed secure direction, in this case $0^{\circ}$, such that at $8^{\circ}$ and $12^{\circ}$, symbols 11 and 01 merge together, severely increasing the BER. Second, in addition to the novel spatial encoding approach presented in this work, the spatial integrity of received QPSK data stream also depends upon the SNR, consequently on the $E_{\mathrm{b}} / N_{0}$ where $E_{\mathrm{b}}$ is the energy per bit. Having said that, our proposed approach can be used for transmitting higher digital modulation schemes like 16 or 64 PSK or Quadrature Amplitude Modulation (QAM). Third, the modulation capability depends primarily on the SP unit, hence any combination of modes can be utilized to scale the proposed system model. In other words, similar results are expected if simultaneous excitation of any two of modes $\{0, \pm 1$, $\pm 2\}$ were to be used. In this paper we have only focused on the use of mode 0 and +1 , conclusions on "which mode combination is the best for a given application?" has not been addressed in this paper. It is worth mentioning that communication scheme with enhanced security in this work is different from the one presented in [33] which shows a directly modulated antenna array when the angular dependence of the signal constellation pattern depends upon the array element spacing. In contrast to this, our approach relies on the simultaneous excitation of all antenna elements and modulation information is translated over the OAM mode mixing transmitter.

Recall Fig. 1, it is assumed that the data stream connected to mode +1 is independently fed to the antenna array and there is no cross-talk between mode 0 and +1 . Contrary to this assumption, mode purity of the OAM transmitter hardware presented in this work is low, as mentioned in section III. As an implication, the expected BER at $0^{\circ}$ is $\sim 10^{-4}$ instead of an ideal 0 . Although the proposed approach works well with the example hardware we used, the received signal BER is expected to improve further by using higher mode purity OAM transmitters. Further information on such OAM beamformers can be found in [34] and the references therein.

Since the transmitted signal carriers arbitrary phase information phase calibration at the receiver end is obtained using a training sequence transmission prior to the data stream. One method of doing this is to first broadcast signal such that SP unit induces a phase shift equal to $-\theta_{\mathrm{PM}}$ for mode \pm 1 and $0.5 \times \theta_{\mathrm{PM}}$ for mode \pm 2 . This will produce a static phase at the receiver antenna even when mode \pm 1 and \pm 2 introduces phase spiral wave-fronts. This training sequence for a given time $T$ is enough to provide a reference phase at the receiver end for a successful calibration. A constant phase ramp at the receiver end is easily detectable since it will follow the same phase ramp pattern of $\Phi_{ \pm 1}$ or $\Phi_{ \pm 2}$ i.e. 0 - $360^{\circ}$ and $0-180^{\circ}$ respectively.

\section{SHORTCOMINGS}

The first shortcoming of the proposed approach is that the performance in terms of data recovery may not be better along the boresight of the OAM beamformer as compare to the directions of higher directivity. This is because of the inherent boresight null positions corresponding to modes +1 and +2 (see Fig. 3). The impact is also visible from the measurement data in Fig. 6 where nulls positioned close to boresight show field almost $25 \mathrm{~dB}$ less than the maximum field, which will have a negative impact on the SNR at a single antenna receiver located at this position. Second, the proposed approach depends critically upon the accuracy of the phase-shifter which can increase the transmitter development cost. Third, the time interval and periodicity of the training sequence will depend upon the channel mobility, and in any case, it will have a cost in terms of additional payload. The proposed approach is suitable for simplex links only. 


\section{CONCLUSION AND FUTURE WORK}

A communication system model with enhanced physical layer security is presented in this work that simultaneously uses two different modes of an OAM beamformer. Information is securely encoded into the mode mixing transmitter when phase modulation is translated in the far-field electric fields. These fields require a single antenna receiver to decode the information after calibrating itself via a training sequence. Results based on measured far-field patterns reveals a BER increase from the order of $10^{-4}$ to $10^{-1}$ when receiver antenna moves $\pm 15^{\circ}$ along elevation from the reference location where the data recovery is the best. The same conclusion is expected to hold valid for any direction, depicting reliable physical layer security. Operational stages of the proposed communication system with enhanced physical layer security using OAM transmitter and a single antenna receiver are summarized in Table I. Detailed investigation on the impact of bandwidth on the quality of the signal received by the single antenna receiver is one of the future directions. Further to this, OAM transmitter hardware complexity reduction requires additional investigation.

\section{ACKNOWLEDGEMENTS}

Authors would like to thank K. Rainey for assistance in the development of the hardware. The work was sponsored by the UK Engineering and Physical Science Research Council EP/P000673/1, EP/N020391/1.

\section{REFERENCES}

[1] M. Chen, L. Jiang, and W. E. I. Sha, "Orbital angular momentum generation and detection by geometric-phase based metasurfaces," Appl. Sci., vol. 8, no. 3, p. 362, 2018.

[2] L. Ma, C. Chen, L. Zhou, S. Jiang, and H. Zhang, "Single-layer transmissive metasurface for generating OAM vortex wave with homogeneous radiation based on the principle of Fabry-Perot cavity," Appl. Phys. Lett., vol. 114, no. 8, p. 81603, 2019.

[3] H. Wang et al., "Vortex beam generated by circular-polarized metasurface reflector antenna," J. Phys. D. Appl. Phys., vol. 52, no. 25, p. $255306,2019$.

[4] J. Wu, Z. Zhang, X. Ren, Z. Huang, and X. Wu, "A Broadband Electronically Mode Reconfigurable Orbital Angular Momentum Metasurface Antenna," IEEE Antennas Wirel. Propag. Lett., 2019.

[5] H.-F. Huang and S.-N. Li, "High-Efficiency Planar Reflectarray With Small-Size for OAM Generation at Microwave Range," IEEE Antennas Wirel. Propag. Lett., vol. 18, no. 3, pp. 432-436, 2019.

[6] K. Bi, J. Xu, D. Yang, Y. Hao, X. Gao, and S. Huang, "Generation of Orbital Angular Momentum Beam With Circular Polarization Ceramic Antenna Array," IEEE Photonics J., vol. 11, no. 2, pp. 1-8, 2019.

[7] C. Guo, X. Zhao, C. Zhu, P. Xu, and Y. Zhang, "An OAM Patch Antenna Design and Its Array for Higher Order OAM Mode Generation," IEEE Antennas Wirel. Propag. Lett., vol. 18, no. 5, pp. 816-820, 2019.

[8] F. Shen, J. Mu, K. Guo, S. Wang, and Z. Guo, "Generation of Continuously Variable-Mode Vortex Electromagnetic Waves With Three-Dimensional Helical Antenna," IEEE Antennas Wirel. Propag. Lett., vol. 18, no. 6, pp. 1091-1095, 2019.

[9] D. Liu, L. Gui, Z. Zhang, H. Chen, G. Song, and T. Jiang, "Multiplexed OAM wave communication with two-OAM-mode antenna systems," IEEE Access, vol. 7, pp. 4160-4166, 2018.

[10] G. Junkin, "A Circularly Polarized Single-Frequency Multimode Helical Beam Antenna," IEEE Trans. Antennas Propag., vol. 67, no. 3, pp. 1459-1466, 2018.

[11] L. Kang, H. Li, J. Zhou, S. Zheng, and S. Gao, "A Mode-Reconfigurable Orbital Angular Momentum Antenna with Simplified Feeding Scheme," IEEE Trans. Antennas Propag., 2019.

[12] C. Xu et al., "Free-space radio communication employing OAM multiplexing based on Rotman lens," IEEE Microw. Wirel. Components
Lett., vol. 26, no. 9, pp. 738-740, 2016.

[13] X.-D. Bai, X.-L. Liang, J.-P. Li, K. Wang, J.-P. Geng, and R.-H. Jin, "Rotman lens-based circular array for generating five-mode OAM radio beams," Sci. Rep., vol. 6, p. 27815, 2016.

[14] W. Park, L. Wang, H.-D. Brüns, D. G. Kam, and C. Schuster, "Introducing a Mixed-Mode Matrix for Investigation of Wireless Communication Related to Orbital Angular Momentum," IEEE Trans. Antennas Propag., vol. 67, no. 3, pp. 1719-1728, 2018.

[15] Y. Yan et al., "High-capacity millimetre-wave communications with orbital angular momentum multiplexing," Nat. Commun., vol. 5, p. 4876, 2014.

[16] B. Allen, A. Tennant, Q. Bai, and E. Chatziantoniou, "Wireless data encoding and decoding using OAM modes," Electron. Lett., vol. 50, no. 3, pp. 232-233, 2014.

[17] D. Lee et al., "An experimental demonstration of $28 \mathrm{GHz}$ band wireless OAM-MIMO (orbital angular momentum multi-input and multi-output) multiplexing," in 2018 IEEE 87th Vehicular Technology Conference (VTC Spring), 2018, pp. 1-5.

[18] Y. H. Cho and W. J. Byun, "Generalized Friis Transmission Equation for Orbital Angular Momentum Radios," IEEE Trans. Antennas Propag., vol. 67, no. 4, pp. 2423-2429, 2019.

[19] Y. Yao, X. Liang, W. Zhu, J. Geng, and R. Jin, "Experiments of Orbital Angular Momentum Phase Properties for Long-Distance Transmission," IEEE Access, vol. 7, pp. 62689-62694, 2019.

[20] M. Klemes, "Reception of OAM radio waves using pseudo-doppler interpolation techniques: A frequency-domain approach," Appl. Sci., vol. 9 , no. 6, p. 1082, 2019.

[21] L. Wang, H. Chen, K. Guo, F. Shen, and Z. Guo, "An Inner-and OuterFed Dual-Arm Archimedean Spiral Antenna for Generating Multiple Orbital Angular Momentum Modes," Electronics, vol. 8, no. 2, p. 251, 2019.

[22] Q. Bai, A. Tennant, and B. Allen, "Experimental circular phased array for generating OAM radio beams," Electron. Lett., vol. 50, no. 20, pp. 1414-1415, 2014.

[23] T. Liu et al., "All-dielectric transformation medium mimicking a broadband converging lens," Opt. Express, vol. 26, no. 16, pp. 2033120341, 2018.

[24] S. Gao, W. Cheng, W. Zhang, and H. Zhang, "Bifocal-Lens Antenna Based OAM Communications System," arXiv Prepr. arXiv1809.04845, 2018.

[25] J. R. Carson, "Notes on the theory of modulation," Proc. Inst. Radio Eng., vol. 10, no. 1, pp. 57-64, 1922.

[26] R. C. Hansen, "Design trades for Rotman lenses," IEEE Trans. Antennas Propag., vol. 39, no. 4, pp. 464-472, 1991.

[27] S. M. Mohammadi et al., "Orbital angular momentum in radio-A system study," IEEE Trans. Antennas Propag., vol. 58, no. 2, pp. 565$572,2009$.

[28] Y. I. Jianjia et al., "Design and validation of a metasurface lens for converging vortex beams," Appl. Phys. Express, 2019.

[29] J. Yi et al., "Design and Validation of an All-Dielectric Metamaterial Medium for Collimating Orbital-Angular-Momentum Vortex Waves at Microwave Frequencies," Phys. Rev. Appl., vol. 12, no. 3, p. 34060, 2019.

[30] L. Torner, J. P. Torres, and S. Carrasco, "Digital spiral imaging," Opt. Express, vol. 13, no. 3, pp. 873-881, 2005.

[31] J. Ren and K. W. Leung, "Generation of High-Purity Millimeter-Wave Orbital Angular Momentum Modes Using Horn Antenna: Theory and Implementation," arXiv Prepr. arXiv1710.00035, 2017.

[32] Y. Rahmat-Samii, V. Galindo-Israel, and R. Mittra, "A plane-polar approach for far-field construction from near-field measurements," IEEE Trans. Antennas Propag., vol. 28, no. 2, pp. 216-230, 1980.

[33] H. Shi and A. Tennant, "Enhancing the security of communication via directly modulated antenna arrays," IET Microwaves, Antennas Propag., vol. 7, no. 8, pp. 606-611, 2013.

[34] Y. Huang et al., "Generation of Broadband High-purity Dual-Mode oAM Beams Using A four-feed patch Antenna: theory and implementation," Sci. Rep., vol. 9, no. 1, pp. 1-10, 2019. 


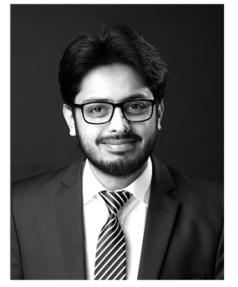

Muhammad Ali Babar Abbasi (S'14-M'17) received the B.S. degree in electrical engineering from CIIT, Islamabad, in 2011, the M.S. degree in electrical engineering from the National University of Sciences and Technology (NUST), Islamabad, Pakistan in 2013, and the Ph.D. degree in electrical engineering from Frederick University, Nicosia, Cyprus, in 2017. From 2017 to 2019, he was Research Fellow with the Centre of Wireless Innovation (CWI), Queen's University Belfast (QUB), Belfast, U.K., where he was involved in finding low-complexity RF front-end solutions for mmWave massive MIMO. He is currently a Lecturer (Assistant Professor) with CWI, QUB. He has authored or co-authored more than 45 journal and conference papers and contributed to 4 book chapters. He was a recipient of the Erasmus Mundus INTACT Doctoral Scholarship by the European Union in 2014. He was the grand prize winner in the Mobile World Scholar Challenge at the Mobile World Congress in 2019 (MWC19). He served as a Reviewer, the Session Chair, and a Technical Program Committee (TPC) member at a number of scientific conferences and workshops.

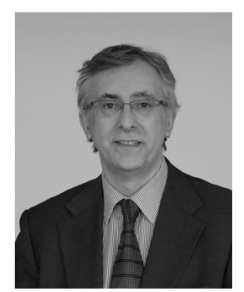

Vincent Fusco (S'82-M82-SM'96-F'04) Research focus on advanced microwave through millimetre wave wireless. Personal Chair of High Frequency Electronic Engineering with QUB. He has authored over 500 scientific papers in major journals and referred international conferences, and two textbooks. He holds patents related to self-tracking antennas and has contributed invited papers and book chapters. His current research interests include physical layer secure active antenna techniques. In 2012, he was awarded the IET Senior Achievement Award, the Mountbatten Medal. He is fellow of the Royal Academy of Engineering.

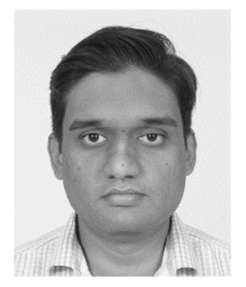

Umair Naeem received his BE degree in electrical engineering from NED University of Engineering and Technology, Karachi, Pakistan, in 2005. He completed his Masters and Doctorate education, with specialization in high frequency electronics and optoelectronics, from University of Limoges, Limoges, France, in 2007 and 2010 respectively. He is currently with Centre for Wireless Innovation, ECIT, Queen's University Belfast, UK. His research interests include synthesis of microwave and millimeter wave components and circuits, multi-functional RF front-ends, active antennas and electromagnetic band gap structures. "

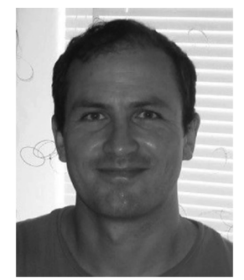

Oleksandr Malyuskin was with the Institute of Electronics, Communications and Information Technology, Queens University Belfast, Belfast, U.K., from 2004 to 2016 . He is currently a Lecturer with the School of Engineering, Ulster University, Newtownabbey, U.K. He has authored over 50 scientific papers in major journals and refereed international conferences. His current research interests include the microwave high-resolution imaging, novel wireless communication techniques, analytical and numerical methods in electromagnetic scattering, time reversal techniques, and biomedical applications.,Dr. Malyuskin was a reviewer for the IEEE publications. 